\title{
Proteasome LMP2/ß1i subunit as biomarker for human uterine leiomyosarcoma
}

\author{
Takuma Hayashi1,10,11, Akiko Horiuchi ${ }^{2}$, Hiroyuki Aburatani ${ }^{3}$, Osamu Ishiko ${ }^{4}$, Nobuo Yaegashi ${ }^{5}$, \\ Yae Kanai ${ }^{6,12}$, Dorit Zharhary7,11, Susumu Tonegawa ${ }^{8}$, Ikuo Konishi ${ }^{9}$ \\ ${ }^{I}$ Department of Immunology and Infectious Disease, Shinshu University School of Medicine, Matsumoto, Nagano, Japan. \\ ${ }^{2}$ Horiuchi Ladies Clinic, Matsumoto, Nagano, Japan. \\ ${ }^{3}$ The Cancer System Laboratory, Research Center for Advanced Science and Technology, The University of Tokyo, Tokyo, Japan. \\ ${ }^{4}$ Department of Obstetrics and Gynecology, Osaka City University Graduate School of Medicine, Osaka, Japan. \\ ${ }^{5}$ Department of Obstetrics and Gynecology, Tohoku University Graduate School of Medicine, Sendai, Miyagi, Japan. \\ ${ }^{6}$ Pathology Division, National Cancer Center Research Institute, Chuoku, Tokyo, Japan. \\ ${ }^{7}$ Sigma-Aldrich Israel Ltd., Rehovot 76100, Israel. \\ ${ }^{8}$ Picower Institution and Department of Biology, Massachusetts Institute of Technology, Cambridge, MA, USA. \\ ${ }^{9}$ Department of Obstetrics and Gynecology, Kyoto University Graduate School of Medicine, Kyoto, Japan. \\ ${ }^{10}$ Promoting Business using Advanced Technology, Japan Science and Technology Agency (JST), Chiyoda, Tokyo, Japan. \\ ${ }^{11}$ SIGMA-Aldrich Collaboration Laboratory. \\ ${ }^{12}$ IHEC and CREST, Japan Science and Technology Agency (JST), Chiyoda, Tokyo, Japan.
}

Received November 27, 2013; Revised December 15, 2013; Accepted January 03, 2014; Published Online January 05, 2014

\section{Scientific $\mathcal{N o t e}$}

\begin{abstract}
Uterine leiomyosarcoma (Ut-LMS) develops more frequently in the myometrium of the uterine body than in the uterine cervix. Although the development of gynecological tumors is often correlated with the secretion of female hormones that of Ut-LMS does not, and its risk factor(s) remain unknown. Importantly, a diagnostic biomarker that can distinguish malignant tumor Ut-LMS from benign tumor leiomyoma (LMA), has yet to be established. Therefore, the risk factor(s) associated with Ut-LMS need to be examined in order to establish a diagnosis and clinical treatment method. Mice with a homozygous deficiency for the proteasome $\beta$-ring subunit, low-molecular mass polypeptide (LMP) $2 / \beta 1$ i spontaneously develop Ut-LMS, with a disease prevalence of $\sim 40 \%$ by 14 months of age. In recent studies, we showed that LMP2/ $\beta 1$ i expression was absent in human Ut-LMS, but present in other human uterine mesenchymal tumors including uterine LMA. Moreover, LMP2/ $\beta 1 \mathrm{i}$ is also known to negatively regulate human Ut-LMS tumorigenesis. Additional experiments furthermore revealed the differential expression of cyclin $\mathrm{E}$ and calponin h1 in human uterine mesenchymal tumors. Therefore, LMP $2 / \beta 1 \mathrm{i}$ is a potential diagnostic biomarker when combined with the candidate molecules, cyclin $\mathrm{E}$ and calponin $\mathrm{h} 1$ for human Ut-LMS, and may be a targeted molecule for a new therapeutic approach.
\end{abstract}

Keywords: LMP2/ß1i; Biomarker; Uterin leiomyosarcoma; Leiomyoma; Proteasome

\section{Scientific Note}

Sarcomas are neoplastic malignancies that typically arise in tissues of mesenchymal origin. The identification of novel molecular mechanisms leading to sarcoma formation and the establishment of new therapies has been hampered by several critical fac-

Corresponding author: Takuma Hayashi; Department of Immunology and Infectious Disease, Shinshu University Graduate School of Medicine, 3-1-1, Asahi, Matsumoto, Nagano 390-8621, Japan. Email: yoyoyo224@hotmail.com

Cite this article as:

Hayashi T, Horiuchi A Aburatani H, Ishiko O, Yaegashi N, Kanai Y, Zharhary D, Tonegawa S, Konishi I. Proteasome LMP2/B1i subunit as biomarker for human uterine leiomyosarcoma. Int $J$ Cancer Ther Oncol 2014; 2(1):02018. DOI: 10.14319/ijcto.0201.8 tors. Human uterine leiomyosarcoma (Ut-LMS) develops more often in the muscle tissue layer of the uterine body than in the uterine cervix. The development of gynecologic tumors is often correlated with female hormone secretion; however, the development of human Ut-LMS is not substantially correlated with hormonal conditions, and the risk factors are not yet known. Importantly, a diagnostic-biomarker which distinguishes malignant Ut-LMS from benign tumor leiomyoma (LMA) is yet to be established. Accordingly, it is necessary to analyze risk factors associated with human Ut-LMS, to establish novel therapeutic method. Proteasome $\beta$-ring subunit LMP2/ $\beta 1$ i-deficient mice spontaneously develop Ut-LMS, with a disease prevalence of $\sim 40 \%$ by 14 months of age. We found LMP2/ $\beta 1$ i expression to be absent in 
human Ut-LMS, but present in human LMA. Therefore, defective-LMP2/ $\beta 1$ i expression may be one of the risk factors for human Ut-LMS. LMP2/ $\beta 1 \mathrm{i}$ is a potential diagnostic biomarker for human Ut-LMS, and may be a targeted-molecule for a new therapeutic approach.

Sarcomas are a rare form of malignant tumor with less than 15,000 new cases diagnosed each year in United States. Though rare, sarcomas are highly debilitating malignancies as they are often associated with significant morbidity and mortality. Sarcomas are biologically very heterogeneous as evidenced by the fact that these malignant tumors arise from a plethora of different tissues and cell types. They are classically defined by their tissue of origin and additionally stratified by their histopathology or patient's age at diagnosis. While most tumors of the uterine body are adenocarcinomas, the uterine cervix tumors are classified into squamous cancer and adenocarcinoma. Uterine mesenchymal tumors, which develop in the myometrium have been traditionally divided into benign LMA and malignant Ut-LMS based on cytological atypia, mitotic activity and other criteria. Ut-LMS is relatively rare, having an estimated annual incidence of 0.64 per 100,000 women. ${ }^{1}$ Ut-LMS accounts for $2 \% \sim 5 \%$ of tumors of the uterine body and develops more often in the muscle layer of the uterine body than in the uterine cervix. As Ut-LMS is resistant to chemotherapy and radiotherapy, surgical intervention is virtually the only means of treatment. ${ }^{2,3}$ The prognosis for Ut-LMS is not good, and the five-year survival rate is approximately $35 \%$. However, developing an efficient adjuvant therapy is expected to improve the survival rate. Uterine LMA may occur in as many as $70 \% \sim 80 \%$ of women by the age of 50 years. ${ }^{4}$ Distinguishing Ut-LMS from other uterine mesenchymal tumors including LMA is very difficult, and a diagnosis generally requires surgery and cytoscopy. Diagnostic categories for uterine mesenchymal tumors and morphological criteria are used to assign cases. The non-standard subtypes of uterine mesenchymal tumors such as the epithelioid and myxoid types are classified in a different way using these features, so the establishment of a diagnostic method for the identification of non-standard smooth muscle differentiation is important. 5,6

High estrogen levels are considered to significantly influence the development of tumors in the uterine body. ${ }^{7}$ The mechanisms by which uterine LMA and Ut-LMS develop are not yet known, though tumors that have developed in the myometrium for some reason gradually become larger due to the influence of the female hormone, estrogen, and generate tumors. However, no correlation between the development of Ut-LMS and hormonal conditions, and no obvious risk factors have been found. Although cases accompanied by hypocalcaemia or eosinophilia have been reported, neither clinical abnormality is an initial risk factor for Ut-LMS. The identification of a risk factor associated with the development of Ut-LMS would significantly contribute to the development of preventive and therapeutic treatments.

Cytoplasmic proteins are mostly degraded by a protease complex, which has many substrates consisting of twenty-eight 20 to 30 $\mathrm{kDa}$ subunits, referred to as the $20 \mathrm{~S}$ proteasome. ${ }^{8,9}$ The proteasomal degradation is essential for many cellular processes, including the cell cycle, the regulation of gene expression and immunological function. ${ }^{10}$ Interferon (IFN)- $\gamma$ induces the expression of large numbers of responsive genes, subunits of proteasome $\beta$-ring, i.e., low-molecular mass polypeptide (LMP) $2 / \beta 1$, LMP7/ $\beta 5$ i, and LMP10/multicatalytic endopeptidase com- plex-like (MECL)-1/ $32 \mathrm{i}^{11}$ A molecular approach to studying the correlation of IFN- $\gamma$ with tumor cell growth has drawn attention. Homozygous mice deficient in LMP2/ $\beta 1$ i show tissue- and substrate-dependent abnormalities in the biological functions of the proteasome. ${ }^{12}$ Ut-LMS reportedly occurred in female LMP2/ $\beta 1$ i-deficient mice at age 6 months or older, and the incidence at 14 months of age was about $40 \% .{ }^{13}$ Histological studies of LMP2/ $\beta$ 1i-lacking uterine tumors have revealed characteristic abnormalities of Ut-LMS. ${ }^{13}$ The tumors consisted of uniform elongated myometrium cells arranged into bundles. The nuclei of the tumor cells varied in size and shape, furthermore, mitosis was frequent. In contrast, the myometrium cells of C57BL/6 mice were normal in appearance. Whereas relatively few ki-67-positive cells, the proliferating cells of solid tumors, were observed in the basal cell layer of the normal myometrium, most of the basal cells vividly expressed ki-67 in LMP2/ $\beta 1$ i-deficient mice. ${ }^{13}$ LMP2/ $/ 1$ i-deficient mice that have developed Ut-LMS undergo considerable weight loss, and then die by 14 months of age. The LMP $2 / \beta 1$ i-deficient mice also exhibit skeletal muscle metastasis from Ut-LMS. Therefore it is likely that LMP $2 / \beta 1$ ideficient mice with Ut-LMS die as a result of the tumor mass and metastasis.

The non-standard subtypes of uterine mesenchymal tumors such as the epithelioid and myxoid types are classified in a different way using these features, so the establishment of a diagnostic method for the identification of non-standard smooth muscle differentiation is important. ${ }^{5,6}$ Pathological studies were performed to demonstrate the validity and reliability of LMP $2 / \beta 1 i$ as a diagnostic biomarker under the combination of other candidate molecules, for instance cyclin E and calponin h1, which reportedly function as anti-oncogenic factor in human Ut-LMS. Pathological examinations revealed a serious loss in the ability to induce LMP2/31i and calponin h1 expressions in human Ut-LMS tissues in comparison with uterine LMA or normal myometrium located in the same section, and markedly cyclin E expression in only human Ut-LMS tissues. ${ }^{14-17}$ Histological findings were consistent with metastatic Ut-LMS for the skeletal muscle and rectum lesions. ${ }^{14,15}$ In western blotting and RT-PCR experiments, LMP2/ $\beta 1$ i was expressed in normal myometrium, but not in human Ut-LMS, both strongly supportive of the pathological findings. ${ }^{14,15,17,18}$ Although we has previously demonstrated that the abnormal expression of the ovarian steroid receptors, Tp53, ki-67 and mutations of Tp53 were frequently associated with Ut-LMS, defective LMP2/ $\beta 1$ i expression appears to be more characteristic of human Ut-LMS than these factors. ${ }^{15,16}$

In the case of gynecological cancers, a female hormonal imbalance is often a risk factor for developing tumors. ${ }^{7}$ As in the case of uterine LMA, however, a correlation between the development of Ut-LMS, the female hormone, and hormone receptors has yet to be elucidated. Recent reports showed the expression of Lmp 2/ $\beta 1 \mathrm{i}$ mRNA and protein in luminal and glandular epitheliua, placenta villi, trophoblastic shells, and arterial endothelial cells. ${ }^{19-21}$ These results implicate LMP2/ $\beta 1 \mathrm{i}$ in the invasion of placental villi, degradation of the extracellular matrix, immune tolerance, glandular secretion, and angiogenesis, but no more information for sarcomagenesis. Further experiments are also required to elucidate the molecular mechanism of human Ut-LMS tumorigenesis involved biological significance of LMP $2 / \beta 1$ i; we are investigating the reliability and characteristics of LMP2/ $\beta 1 \mathrm{i}$ as a diagnostic indicator with several clinical research facilities. Histopathologic characteristics of human uterine 
mesenchymal tumors including mitotically active leiomyoma, bizarre leiomyoma, lipoleiomyoma, undifferentiated endometrial sarcoma, epithelioid variant leiomyosarcoma, myxoid variant leiomyosarcoma, smooth muscle tumors of uncertain malignant potential (STUMP), leiomyomatoid angiomatous neuroendocrin tumor (LANT) are summarized. ${ }^{22,23}$ Clarification of the correlation between these factors and the development of human Ut-LMS and the identification of specific risk factors may lead to the development of new clinical treatments for the disease.

\section{Acknowledgements}

This study was supported in part by grants from the Ministry of Education, Culture, Science and Technology, and The Foundation of Osaka Cancer Research, and The foundation for the Promotion of Cancer Research, The Kanzawa Medical Research Foundation and The Takeda Foundation for Medical Science

\section{Conflict of interest}

The authors declare that they have no conflicts of interest. The authors alone are responsible for the content and writing of the paper.

\section{References}

1. Zaloudek C, Hendrickson MR. Mesenchymal tumors of the uterus, in Kurman RJ.(ed): Blaustein`s Pathology of the Female Genital Tract (ed 5). New York, Springer-Verlag 2002; 5: 561-78.

2. Wu TI, Chang TC, Hsueh S, Hsu KH, Chou HH, Huang HJ, Lai $\mathrm{CH}$. Prognostic factors and impact of adjuvant chemotherapy for uterine leiomyosarcoma. Gynecol Oncol 2006; 100: 166-72.

3. Leitao MM, Soslow RA, Nonaka D, Olshen AB, Aghajanian C, Sabbatini P, Dupont J, Hensley M, Sonoda Y, Barakat RR, Anderson S. Tissue microarray immunohisto chemical expression of estrogen, progesterone, and androgen receptors in uterine leiomyomata and leiomyosarcoma. Cancer 2004; 101: 1455-62.

4. http://cancer.gov/cancertopics/pdq/treatment/uterinesar coma/HealthProfessional (Accessed on December 15, 2013)

5. Kurma RJ. Pathology of the Female Genital Tract, 4th ed. New York, Springer-Verlag 2001; 4: 499.

6. Diagnostic Criteria for LMS, Adapted from $2003 \mathrm{WHO}$ Guidelines: (2003) World Health Organization Classification of Tumours: Pathology and Genetics, Pathology and Genetics of Tumours of the Breast and Female Genital Organs. IARC Press, France.

7. Lin JF, Slomovitz BM. Uterine sarcoma. Curr Oncol Rep 2008; 10: 512-8.

8. Peters JM, Franke WW, Kleinschmidt JA. Distinct $19 \mathrm{~S}$ and $20 \mathrm{~S}$ subcomplexes of the $26 \mathrm{~S}$ proteasome and their distribution in the nucleus and the cytoplasm. $J$ Biol Chem 1994; 269: 7709-18.

9. Lodish H, Berk A, Matsudaira P, Kaiser CA, Krieger M, Scott MP, Zipursky SL, Darnell J. 2004 "3". Mol Cell Biol (5th ed.). New York: W.H. Freeman and CO. 2004; 5: 66-72.
10. Konstantinova IM, Tsimokha AS, Mittenberg AG. Role of proteasomes in cellular regulation. Intl Rev Cell Mol Biol 2008; 267: 59-124.

11. Wang J, Maldonado MA. The Ubiquitin-Proteasome System and Its Role in Inflammatory and Autoimmune Diseases. Cell Mol Immunol 2006; 3: 255-61.

12. Van Kaer L, Ashton-Rickardt PG, Eichelberger M, Gaczynska M, Nagashima K, Rock KL, Goldberg AL, Doherty PC, Tonegawa S. Altered peptidase and viral-specifi c $\mathrm{T}$ cell response in LMP2 mutant mice. Immunity 1994; 1: 533-41.

13. Hayashi T, Faustman DL. Development of spontaneous uterine tumors in low molecular mass polypeptide-2 knockout mice. Cancer Res 2002; 62: 24-7.

14. Hayashi T, Kobayashi Y, Kohsaka S, Sano K. The mutation in the ATP-binding region of JAK1, identified in human uterine leiomyosarcomas, results in defective interferon-gamma inducibility of TAP1 and LMP2. Oncogene 2006; 25: 4016-26.

15. Hayashi T, Horiuchi A, Sano K, Hiraoka N, Kasai M, Ichimura T, Nagase S, Ishiko O, Kanai Y, Yaegashi N, Aburatani H, Shiozawa T, Tonegawa S, Konishi I. Potential role of LMP2 as tumor-suppressor defines new targets for uterine leiomyosarcoma therapy. Sci Rep 2011; 1:180.

16. Zhai YL, Kobayashi Y, Mori A, Orii A, Nikaido T, Konishi I, Fujii S. Expression of steroid receptors, $\mathrm{Ki}-67$, and p53 in uterine leiomyosarcomas. Intl $J$ Gynecol Pathol 1999; 18: 20-8.

17. Hayashi T, Horiuchi A, Sano K, Hiraoka N, Kasai M, Ichimura T, Nagase S, Ishiko O, Kanai Y, Yaegashi N, Aburatani H, Shiozawa T, Tonegawa S, Konishi I. Potential role of LMP2 as an anti-oncogenic factor in human uterine leiomyosarcoma: morphological significance of calponin h1. FEBS Letter 2012; 586: 1824-31.

18. Hayashi T, Horiuchi A, Konishi I. Tumor growth arrest: involvement of the mutation in the cataritic region of JAK1. European Journal of Research in Medical Science 2013; 1: 8-21.

19. Wang HX, Wang HM, Li QL, Lin HY, Qian D, Zhu C. Expression of proteasome subunits low molecular mass polypeptide (LMP) 2 and LMP7 in the endometrium and placenta of rhesus monkey (Macaca mulatta) during early pregnancy. Biol Reprod 2004; 71: 1317-1324.

20. Wang HX, Wang HM, Lin HY, Yang Q, Zhang H, Tsang BK, Zhu C. Proteasome subunit LMP2 is required for matrix metalloproteinase-2 and -9 expression and activities in human invasive extravillous trophoblast cell line. J Cell Physiol 2006; 206: 616-23.

21. Fu JJ, Lin P, Lv XY, Yan XJ, Wang HX, Zhu C, Tsang BK, Yu XG, Wang H. Low molecular mass polypeptide-2 in human trophoblast: over-expression in hydatidiform moles and possible role in trophoblast cell invasion. Placenta 2009; 30: 305-12.

22. Sakashita N, Yamada M, Nakagawa T, Yamasaki H, Takeya M. A leiomyomatoid angiomatous neuroendocrine tumor of the myometrium: case study with ultrastructural analysis. Hum Pathol2008; 39: 788-92.

23. Avritscher R, Iyer1 RB, Ro J, Whitman G. Lipoleiomyoma of the Uterus. AJR Am J Radiol 2001; 177: 856. 